\title{
Switched Unfalsified Multicontroller
}

\author{
Fernando Costa, Fernando Coito, and Luís Palma \\ Departamento de Engenharia Electrotécnica, \\ Faculdade de Ciências e Tecnologia, Universidade Nova de Lisboa, \\ 2829-516 Caparica, Portugal \\ $\{1 \mathrm{bp}, \mathrm{fjvc}\}$ afct.unl.pt
}

\begin{abstract}
In this paper, we present a controller design strategy for the implementation of a multicontroller structure for single-input single-output (SISO) plants. The overall control system can be viewed as a feedback interconnection of a SISO plant, a set of candidate controllers and a switched selection scheme that supervises the switching process among the candidate controllers. The switching scheme is designed without explicit assumptions on the plant model, based on the unfalsified control concept introduced by Safonov et al. [1, 2]. A switched multicontroller structure is implemented and experimental results are presented.
\end{abstract}

Keywords: multiple model control, adaptive control, switched control.

\section{Introduction}

Dealing with nonlinear systems is an inherently difficult problem. As a consequence models and analysis of nonlinear systems will be less precise than for the simpler linear case. Thus, one should look for model representations and tools that utilize less precise system knowledge than the traditional approaches. This is indeed the trend in the area of intelligent control where a range of approaches, such as Fuzzy Logic, Neural Networks and Probabilistic Reasoning are being explored [3]. The current paper uses operating regime decomposition for the partitioning of the operating range of the system in order to solve modeling and control problems.

\subsection{Unfalsified Switching Control}

The operating regime approach leads to multiple-model or multiple controller (multiple model control - MMC) synthesis, where different local models/controllers are applied under different operating conditions, see Fig. 1. One version of the above strategy is to represent the global system as a family of smaller local regions, where the supervisory controller alters the controller according to the current local region in which the process is operating. It must be stressed that this strategy holds only if the nonlinear system can be represented as a Linear Parameter varying (LPV) system.

The switching is orchestrated by a specially designed logic that uses the measurements to assess the performance of the candidate controller currently in use and also the potential performance of alternative controllers. In performance-based supervision 


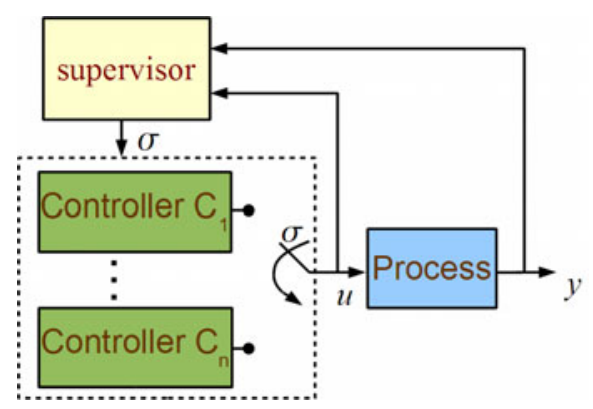

Fig. 1. Switching control. The switching decision between the controllers is performed trough the switching signal $\sigma$.

the supervisor attempts to assess directly the potential performance of every candidate controller, without estimating the model of the process $[1,2,4]$. To achieve this, the supervisor computes performance signals that provide a measure of how well the controller $C_{i}$ would perform in a conceptual experiment, in which the actual control signal $u$ would be generated by $C_{i}$ as a response to the measured process output $y$. This approach is inspired by the idea of controller unfalsification [1].

Using the unfalsification concept, no assumptions on the plant structure are required. The best controller among a set of candidate is selected straight from input/output data. The performance of all candidate controllers is evaluated directly, at every time instant, without actually inserting them in the feedback loop. Controllers that prove to be unable to drive the system according to the desired closed loop dynamics are entitled falsified. Only unfalsified controllers are candidate to actually control the process. Thus, switching between candidate controllers is based directly on their performance.

\subsection{Controller Design}

A key feature over the unfalsified control approach is the separation between the supervisor switching policy, and the controllers design and tuning procedure. Apart for some causality constrains, there are no relevant restrictions on individual controller structures. In fact, different controller structures may be combined into a single unfalsified switched multicontroller.

A relevant aspect on unfsalsified control is the fact that, in spite no process model is required for the development of the supervisor switching scheme, all the controllers share the same closed loop specifications, usually in the form of the behavior from a reference model to be tracked. This makes its use within muti-loop control structures quite interesting, as it provides a level of decoupling between loop dynamics and the process operation conditions.

Within this framework two different approaches towards the development of such a multicontroller were developed. The first applies a set of standard state space based pole placement controllers, using Kalman filter for state estimation. The second uses non-parametric process models and the set of controllers is determined from experimental frequency response data, through frequency domain optimization. Due to space constrains this second controller will be further described in a latter article. 


\section{Contribution to Sustainability}

The keyword that is always tied to control, even when it is not explicitly mentioned, is "performance". Within the control field performance may be evaluated by a broad spectrum of index functions, however, in practical applications the ultimate performance assessment is related to the quality of the process outcome and the efficient use of resources - materials, energy and time. Both resources and quality are essential topics for sustainability.

\section{The Unfalsified Pole Placement Multicontroller}

There is vast literature on supervisory control, mainly for process estimation based schemes. Among those based in process estimation using Certainty Equivalence, interesting references are $[6,7,8,9]$, while for or Model Validation based schemes some relevant papers are $[10,11]$. Also, a very interesting tutorial may be found in [5] where an attempt is made to integrate the different approaches within a unified framework.

As for performance evaluation based algorithms, and specially unfalsified control, some important references are $[1,2,4,12]$.

It is well known that switching among stabilizing controllers can easily result in an unstable system [9, 47]. To avoid a possible loss of stability caused by switching one should then require the switching logic to prevent "too much" switching, by implementing a dwell-time strategy $[13,4]$.

\subsection{Unfalsified Controllers}

Consider that the process to be controlled is unknown and that the only available information is the past values from the set-point $(r)$, the output $(y)$ and the control action $(u)$. The aim is to determine if a controller is capable to lead the closed loop system to behave according to some predefined reference model $W_{m}$.

It is assumed that there is a number of predesigned "causally-left-invertible" controllers $C_{i}$ (in the sense that the current value of $r_{i}(t)$ is uniquely determined by past values of $u(t)$ and $y(t)$ ), among which at least one is able to fulfill the specification. After Safonov [1] performance criterion (1) is used to evaluate discrete time controllers.

$$
V(\tilde{r}, u, \tilde{e}, t)=\left\{\begin{array}{ccc}
\frac{\|\tilde{e}\|_{t}+\lambda\|u\|_{t}}{\|\tilde{r}\|_{t}} & \text { if } & \|\tilde{r}\|_{t} \neq 0 \\
\infty & \text { if } & \|\tilde{r}\|_{t}=0 \mathrm{e}\|\tilde{e}\|_{t}+\lambda\|u\|_{t} \neq 0 \\
0 & \text { if } & \|\tilde{e}\|_{t}+\lambda\left\|_{t}\right\|_{t}=0
\end{array}\right.
$$

were $\lambda$ is a parameter $(>0)$ and the norm is $\|x\|_{t}=\sqrt{\sum_{0}^{t} \rho^{\tau} x^{T}(\tau) x(\tau)} ; \rho$ is a used a an exponential forgetting factor $(<1)$. 
At every moment, the controller performance is evaluated for all $C_{i}$ according to the procedure (see also Fig. 2):

1. The plant is to be under control of a stabilizing controller, even if its performance is poor.

2. From past input/output data compute a fictitious set-point signal $\tilde{r}_{i}(t)=\tilde{r}_{i}\left(C_{i},\left.u(\tau)\right|_{\tau \leq t},\left.y(\tau)\right|_{\tau \leq t}\right)$ for each controller $C_{i}$. This corresponds to the set-point signal for which, taking into account $y(t)$, the controller would have produced the actual control action $u(t)$.

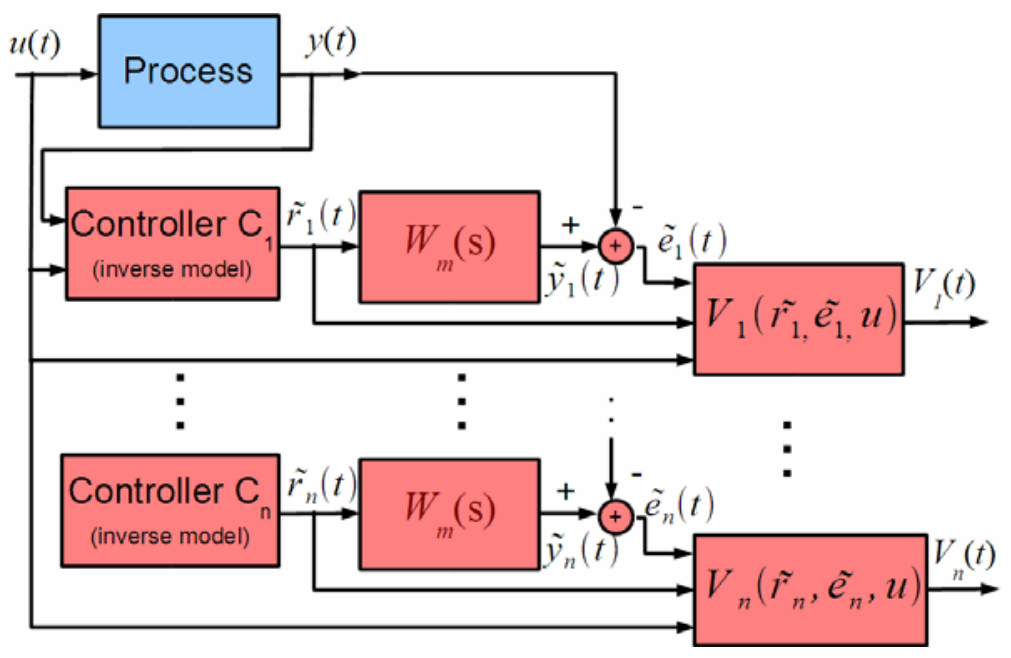

Fig. 2. Controller performance evaluation under unfalsified control framework

3. For each controller compute the fictitious output signal $\tilde{y}(t)$, corresponding to the output of the reference model $W_{m}$, when the fictitious set-point signal $\tilde{r}_{i}(t)$ is used.

4. For each controller compute the fictitious error $\tilde{e}_{i}(t)=\tilde{y}_{i}(t)-y_{i}(t)$.

5. For each controller evaluate the performance function $V(\tilde{r}, u, \tilde{e}, t)$.

6. From controller $C_{i}$ performance $V\left(\tilde{r}_{i}, u, \tilde{e}_{i}, t\right)$ together with a performance threshold $\gamma$, the controller is said to be falsified by the available data at time $t$, if $V\left(\tilde{r}_{i}, u, \tilde{e}_{i}, t\right)>\gamma$.

\subsection{Switch Limiting Strategy}

Only unfalsified controllers are candidates to control the process, which means that each individual controller yields a stable closed loop system. The basic control selection approach is to choose the controller with the least performance index. However, this may raise stability problems, resulting from fast switching of the active controller $[9,7]$. Thus some switch limiting strategy is required. The most common solution is 
the use of a dwell-time. However, two other switching policies are frequently used with unfalsified control switching schemes:

- Once controller $C_{i}$ is chosen, stick to this controller even though it may not be the best. Controller switching occurs only when $V\left(\tilde{r}_{i}, u, \tilde{e}_{i}, t\right)$ rises above a threshold, at which time the controller with the least performance index ought to be chosen.

- Another strategy is to define a switching offset. In this case when controller $C_{i}$ is in use, no switching takes place as long as no other controller performance index lies below $V\left(\tilde{r}_{i}, u, \tilde{e}_{i}, t\right)-\gamma_{s}$. This is the policy used in the results from this paper.

\subsection{Pole Placement Control Design}

A relevant feature under the unfalsified control approach is independence from the controller algorithm. As long as the process is working in stable close loop system, it is possible to evaluate each individual controller performance, even though it is not the actual active controller. This allows the used of any causally-left-invertible linear time invariant controllers.

For pole placement design a set of state space models is required (2), representing the process dynamics over the relevant range of operating conditions. This may be obtained using standard identification methods. The spread of models over the operating range is not critical, as long as it adequately captures the full range.

$$
\begin{aligned}
& \mathbf{x}_{i}(k)=\mathbf{A}_{i} \mathbf{x}_{i}(k-1)+\mathbf{B}_{i} u(k-1) \\
& y(k)=\mathbf{C}_{i} \mathbf{x}_{i}(k)+D_{i} u(k)
\end{aligned}
$$

For each model a controller is design. The controller structure is

$$
u_{i}(k)=-\mathbf{K}_{i} \hat{\mathbf{x}}_{i}(k)+N_{i} r(k) \quad i=1, \ldots, n
$$

where $\hat{\mathbf{x}}_{\mathbf{i}}(k)$ is the output from a state estimator, $r(k)$ is the set-point, $N_{\mathbf{i}}$ is a parameter and $\mathbf{K}_{\mathbf{i}}$ is a parameter vector. Each controller is designed so that the closed loop system will behave according to some specified dynamics (the same for all the model/controller).

\subsection{Frequency Optimization Based Design}

With low noise processes, by using experimental frequency response to characterize the dynamic behavior over the selected operating points it is possible to obtain models (non-parametric) that are closer to the process true behavior. From such models controllers may be designed by classical frequency based methods (Nyquist plot, lead-lag compensation, etc.), or by optimization over the frequency based algorithms. An interesting methodology for this purpose is presented in [14], where a two-stage optimization scheme is used. 


\section{Implementation and Test}

Unfalsified multicontroller supervisors were implemented and tested, using both of the proposed structure and design approaches. This section addresses some implementation issues and experimental results. Tests are made over a lab-scale heat/ventilation experiment (Fig. 3). Three operation regimes are defined according to fan speed: low, medium and high speed. Fig. 3. also shows the frequency response corresponding to each of this regimes.

Fig. 3.b shows that at low frequencies the process gain decreases with the fan speed. The process bandwidth increases with the speed. The phase plot shows that the process presents some transport time delay.

\subsection{Unfalsified Pole Placement Switched Controller}

For each of the selected operation regime a linear second order state space model (2) is identified. Fig. 4 shows a comparison between the process experimental frequency

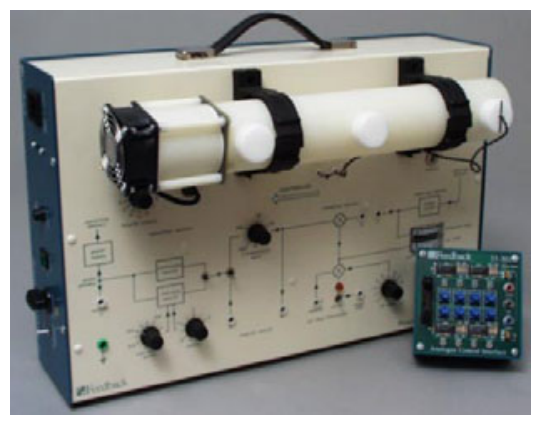

(a)
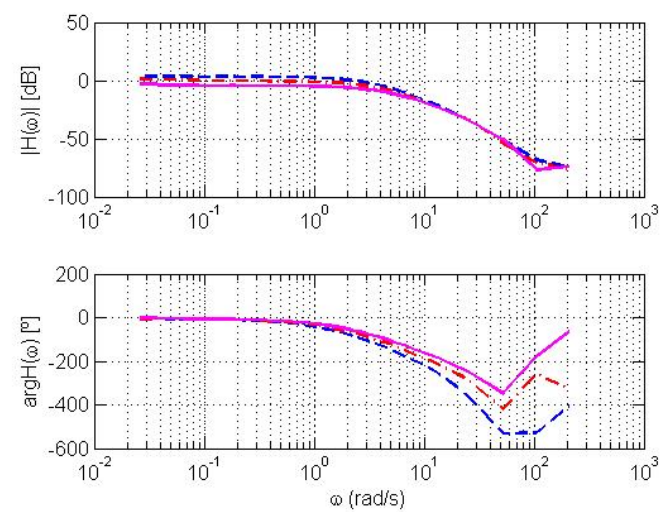

(b)

Fig. 3. a) Lab-scale heat/ventilation experiment used for tests. b) Process frequency response for low speed (---blue), medium speed (---red) and high speed (-magenta). 
response an that of the identified model. The models gain present a small deviation for low frequencies, but in the overall captures the plant behavior for values above $-20 \mathrm{~dB}$.

The models phase captures the systems behavior at lower frequencies. However the process transport delay is not captured by the model leading to an increasing difference at higher frequencies. Nevertheless, the model is found to capture the fundamental of the process behavior over the relevant dynamic range.
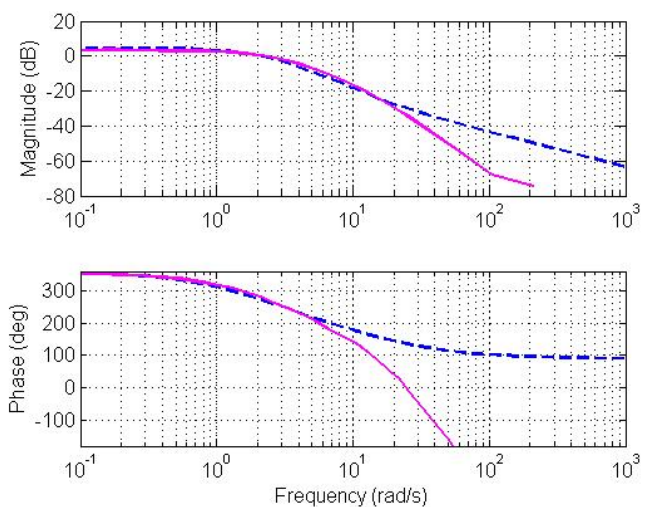

Fig. 4. Process frequency response for low speed (- magenta) and the corresponding state space model frequency response (---blue)

Pole placement controllers (3) are designed according to the closed loop reference model specifications. These are defined in terms of its step response: i) $3 \%$ overshoot; ii) settling time of 1 second; iii) zero steady state tracking error. This leads to a reference model represented by the transfer function

$$
Y(s)=\frac{16}{s^{2}+6 s+16} R(s)
$$

Experimental tests show that the proposed controller structure (3), combined with the identified models, is able to fulfill specifications i) and ii), but yields a significant steady state error. This results from the low frequency modeling error. Thus the control structure from Fig. 1 is adapted to include integral control action (see Fig. 5).

As under unfalsified all the controllers share the same reference model, a shared integral action may be used. If the individual controllers were tuned for different specification sets, it would be necessary to tune a separate control action for each controller.

Fig. 6 illustrates the use of the proposed multicontroller structure applied to the lab-experiment. The test starts at medium fan speed, close to 55 seconds the speed changes to low, and at 105 seconds it changes to high.

The process output follows closely the set-point, with no relevant overshoot and with fast set-point transitions. Operating conditions changes cause disturbances on the output signal that are rapidly recovered. The second fan speed change causes a large spike on the output signal, but it must be stressed that it corresponds to a change from the lower to the higher fan speed. This causes a very large modification on the process gain. 


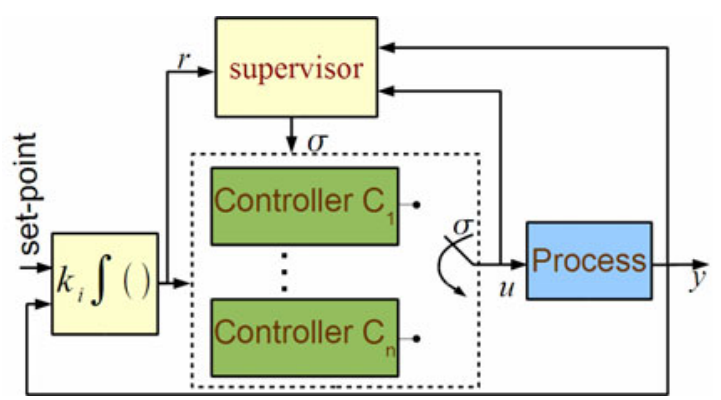

Fig. 5. Supervisory control structure with shared integral action
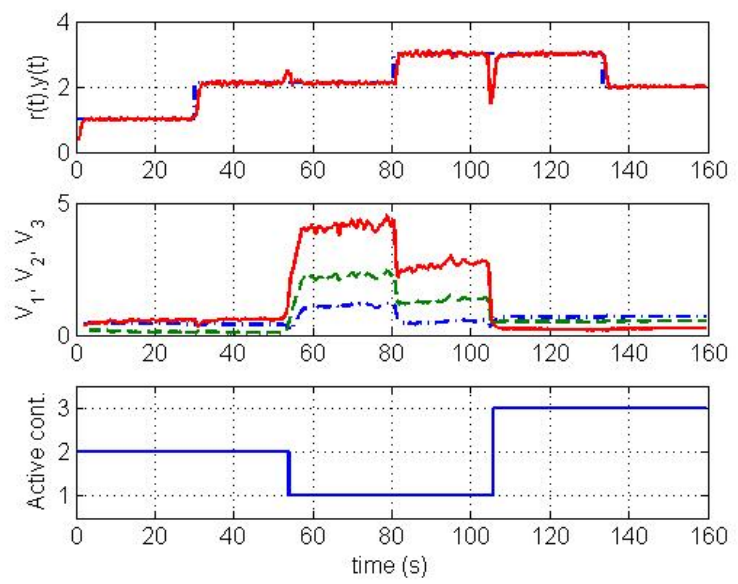

Fig. 6. Supervisory control test over the experimental setup. $r(t)$ - set-point; $y(t)$ - output; $V_{1}$, $V_{2}, V_{3}$ - performance indexes. Active action: 1 - low speed; 2 - medium speed; 3 - high speed.

Observing the performance indexes $\left(V_{1}, V_{2}, V_{3}\right)$ it is apparent that the correct controller is selected in all the operating conditions. Operating conditions changes are rapidly detected.

\section{Conclusions and Further Work}

The switched multicontroller structure described shows to present good performance and fast adaptation to modifications on the operating conditions.

An important feature is that no previous knowledge on the plant dynamics is required to implement the unfalsified control-switching scheme. As the performance evaluation algorithm does not require a specific controller structure, it can be used with a broad range of controllers, and its possible to combine controllers of different types into a single switched multicontroller. 
Once the control-switching scheme requires no process model, an interesting development is to design the controllers without using any process parametric models. As mentioned in the paper, this may be achieved through the use of experimental frequency response to characterize the dynamic behavior over the operating range.

\section{References}

1. Safonov, M.G., Tsao, T.-C.: The Unfalsified Control Concept and Learning. IEEE Trans. Aut. Cont. 42, 843-847 (1997)

2. Safonov, M.G., Cabral, F.B.: Fitting controllers to data. Syst. \& Cont. Letters 43, 299-308 (2001)

3. Palma, L.B., Coito, F.J., Neves-Silva, R.A.: Robust Fault Diagnosis Approach using Analytical and Knowledge-Based Techniques Applied to a Water Tank System. Int. J. Eng. Int. Syst. for Elect. Eng. and Comm. 13, 237-244 (2005)

4. Wang, R., Safonov, M.G.: Stability of Unfalsified Adaptive Control. In: 2005 American Control Conference, pp. 3162-3167 (2005)

5. Hespanha, J.P.: Tutorial on supervisory control. Technical report, Dept. of Electrical and Computer Eng., University of California, Santa Barbara (2001)

6. Morse, A.S.: Supervisory control of families of linear set-point controllers-part 1: exact matching. IEEE Trans. on Automat. Contr. 41, 1413-1431 (1996)

7. Morse, A.S.: Supervisory control of families of linear set-point controllers-part 2: robustness. IEEE Trans. on Automat. Contr. 42, 1500-1515 (1997)

8. Narendra, K.S., Balakrishnan, J.: Adaptive control using multiple models. IEEE Trans. on Automat. Contr. 42, 171-187 (1997)

9. Hespanha, J.P., Morse, A.S.: Certainty equivalence implies detectability. Syst. \& Contr. Lett. 36, 1-13 (1999)

10. Kosut, R., Lau, M., Boyd, S.: Set-membership identification of systems with parametric and nonparametric uncertainty. IEEE Trans. on Automat. Contr. 37, 929-941 (1992)

11. Kosut, R.: Uncertainty model unfalsification: A system identification paradigm compatible with robust control design. In: Proc. of the 34th Conf. on Decision and Contr., pp. 3492 3497 (1995)

12. van Helvoort, J., de Jager, B., Steinbuch, M.: Data-driven multivariable controller design using Ellipsoidal Unfalsified Control. Syst. \& Cont. Letters 57, 759-762 (2008)

13. Liberzon, D., Morse, A.S.: Basic problems in stability and design of switched systems. IEEE Contr. Syst. Mag. 19, 59-70 (1999)

14. Coito, F.J., Ortigueira, M.D.: Fractional Controller Design Trough Multi-Objective Optimization. In: 8th Portuguese Conf. on Aut. Cont. - CONTROLO 2008 (2008) 